\title{
Glimpsing the Compositions of Sub-Neptune-Size Exoplanets
}

\author{
Leslie A. Rogers \\ Hubble Fellow, California Institute of Technology, \\ 2100 E. California Blvd., MC 249-17, Pasadena, USA, 91125 \\ email: larogers@caltech.edu
}

\begin{abstract}
The growing number of transiting planets with mass constraints opens the possibility of applying a statistical approach to learn about the underlying population of planet compositions. We focus on the intriguing transition between rocky exoplanets and planets with voluminous gas layers, and explore how the current census of sub-Neptune-size exoplanets constrains the maximum radii of rocky planets. We outline a hierarchical Bayesian model approach to infer the fraction of planets that are dense enough to be rocky (as a function of planet radius). A preliminary analysis of the current sample of planets with mass and radius constraints reveals that most planets larger than $1.9 R_{\oplus}$ are too low density to be comprised of Fe and silicates alone.
\end{abstract}

Keywords. (stars:) planetary systems, (stars:) planetary systems: formation

\section{Introduction}

Exoplanets detected both in transit and by their dynamical influence are very valuable. The radius derived from the transit depth and the mass derived from radial velocity (RV) measurements or transit timing variations, together give the planet density and some handle on the planet composition. To date more than 190 transiting planets have measured masses (e.g. exoplanets.org). The accumulating census of transiting planets with measured masses contains information about the underlying composition distribution of planets, and about the masses of the more than 3000 Kepler transiting planet candidates (Borucki et al. 2011, Batalha et al. 2013) that lack dynamical confirmation.

Planet mass-radius measurements can be especially informative for planets that are smaller than Neptune $\left(R_{p} \lesssim 4 R_{\oplus}\right)$. For planets in this size range, a wide diversity of planet compositions are a priori plausible; rock, astrophysical ices $\left(\mathrm{H}_{2} \mathrm{O}, \mathrm{NH}_{3}\right.$, and $\left.\mathrm{CO}\right)$, and $\mathrm{H} / \mathrm{He}$ gas can all make significant contributions to both the planets' mass and volume (e.g., Rogers \& Seager 2010a, 2010b). In the Solar System, there are no planets with masses and radii intermediate between the Earth and the ice giants. As a statistical sample of sub-Neptune-sized exoplanets with measured masses and radii accumulates, we may begin to study the intriguing transition between planets with rocky compositions and those with significant contributions from volatiles (astrophysical ices and $\mathrm{H} / \mathrm{He}$ ).

The Kepler Mission has already made a tremendous contribution to populating the planet mass-radius diagram (Figure 1), and more sub-Neptune-sized Kepler transiting planets with mass constraints will soon be announced. The Kepler team undertook an ambitious follow-up program with Keck-HIRES to measure the radial velocities of 22 Kepler Objects of Interest (KOIs) hosting planet candidates with $R_{p}<4 R_{\oplus}$ (Marcy et al. 2013, in prep). The planet candidates were selected for RV follow-up based on their quiet host stars (Kepler Magnitude $K_{p}<13.5, T_{\text {eff }}<6100 \mathrm{~K}, v \sin i<5 \mathrm{~km} \mathrm{~s}^{-1}$ ) and detectable predicted RV-amplitudes $\left(K>1 \mathrm{~m} \mathrm{~s}^{-1}\right)$. Each target received 20 to 50 
RV measurements between July 2009 and May 2013. This large follow-up effort will more than double the number of known sub-Neptune-size transiting planets with mass constraints, yielding mass measurements or upper limits for 52 planets (42 transiting and 10 non-transiting) in 22 planetary systems.

With the release of the new Marcy et al. (2013) sample of sub-Neptune-size transiting planets with RV follow-up, we can start to move beyond case studies of individual objects towards statistical studies of the population of planets. One trend that is already readily visible in Figure 1 is a high density - high mass threshold among the planet mass-radius pairs measured to date. In the rest of this paper, we focus on this apparent paucity of massive rocky planets in greater detail, and set statistical constraints on the fraction of planets that are dense enough to have rocky compositions as a function of planet radius. We identify which planets are dense enough to be rocky (Section 2), and outline a statistical approach to studying the distribution of planet compositions (Section 3).

\section{Which planets may be rocky?}

One of the most direct insights we can glean about a sub-Neptune exoplanet's composition is whether it must have some volatiles $(\mathrm{H} / \mathrm{He}$ or astrophysical ices). The mass-radius relation for a silicate composition (the solid curve in Figure 1) represents an extreme lower limit to the masses of rocky planets with no volatiles $\left(M \operatorname{rocky}, \min \left(R_{p}\right)\right)$. Planets that are less dense must have some volatiles (in the form of water or $\mathrm{H} / \mathrm{He}$ ); though rock may still account for much of their mass, these planets have mean densities that are too low for their transit radius defined by a rocky surface. Planets that are more dense than $M$ rocky, $\min \left(R_{p}\right)$ could potentially be comprised just of iron and silicates.

We must account for the large measurement uncertainties on planet masses and radii when assessing which planets may be rocky. Many of the Kepler planets with RV followup have either marginal RV detections or mass upper limits, yet even the non-detections contain information about the planet composition. With that aim, we introduce $p_{\text {rocky }}$, the posterior probability that a planet is dense enough to be rocky based on the measured mass and radius. $p_{\text {rocky }}$ is evaluated as the fraction of a transiting planets' joint
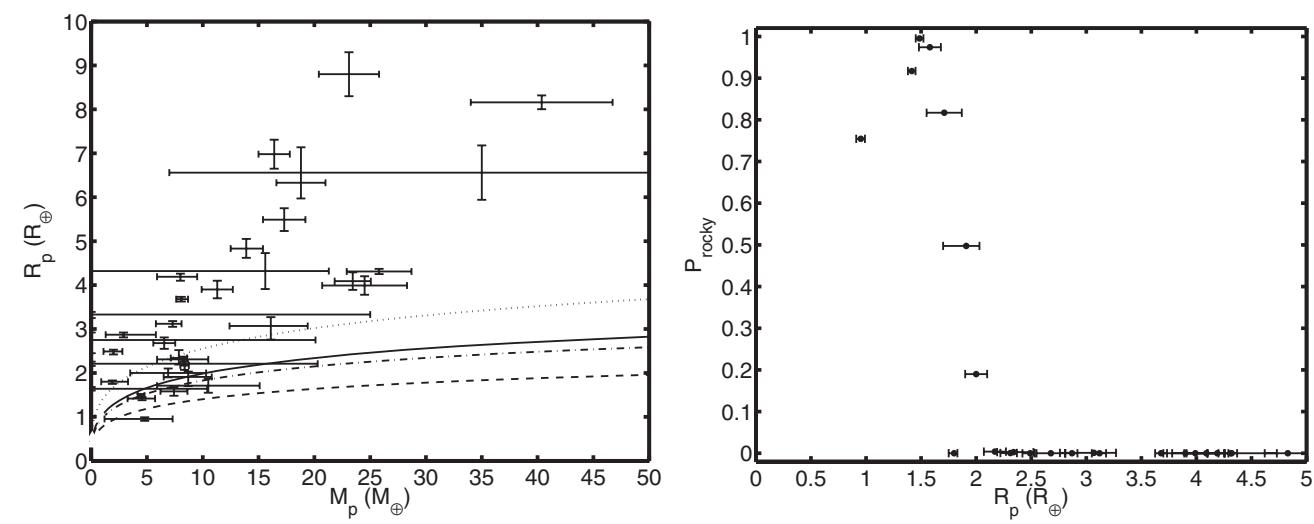

Figure 1. Left: Exoplanet Mass-Radius Diagram. The curves are theoretical mass-radius relations for a fixed planet composition from Seager et al. (2007). The dashed, dot-dashed, solid, and dotted curves are for hypothetical pure iron, Earth-like composition $(32 \% \mathrm{Fe}, 68 \%$ silicate by mass), pure silicate, and pure water ice planets, respectively. Right: The posterior probability that a planet is dense enough to be rocky, $p_{\text {rocky }}$, as a function of the planet radius. Planets with $p_{\text {rocky }}>0.1$ include Kepler-68c, Kepler-10b, Kepler-36b, CoRoT-7b, KOI-94b, Kepler-20b, and Kepler-18b (in order of increasing radius). 
mass-radius posterior probability density (obtained from fitting a planet model to the radial velocity data and transit light curve) that falls within the high-density potentially rocky regime $\left(M_{p} \geqslant M\right.$ rocky, $\left.\min \left(R_{p}\right)\right)$. Planets with $p_{\text {rocky }}$ near 1 are well constrained to have masses and radii in the potentially rocky regime, while low-density planets have $p_{\text {rocky }}$ near 0. RV Marginal detections or non-detections, may have measured RV amplitudes that spill into unphysical regimes (corresponding to negative mass or masses exceeding that of a pure iron sphere). In calculating $p_{\text {rocky }}$, we assume a flat prior on planet mass-radius pairs that are physically plausible.

The values of $p_{\text {rocky }}$ for all known transiting planets with mass constraints are presented in Figure 1. We find that smaller planets are more likely to be dense enough to be rocky. Planets larger than $\sim 2 R_{\oplus}$ are too low density to be comprised of iron and silicates alone, there is some sort of transition regime between 1.5 and $2 R_{\oplus}$.

\section{Distribution of planet Compositions}

We now take our analysis one step further to evaluate what can be inferred about the composition distribution of the underlying population of planets. We present a statistical approach to constrain the fraction of planets that are dense enough to be rocky (as a function of planet radius). A population-level analysis such as this, will be especially warranted once the Marcy et al. (2013) RV mass constraints are released. Here, for demonstration, we apply our approach to the current list of planets with mass and radius constraints from exoplanets.org (Wright et al. 2011). We emphasize, however, that the exoplanets.org list of transiting planets with mass constraints is an inhomogeneous sample of planets, and is subject to selection biases. We do not directly account for selection effects here, but they will be a priority for future work.

We take a Bayesian approach to investigate how the fraction of planets that are dense enough to be rocky depends on planet radius. We begin by assuming some simple functional form for $f_{\text {rocky }}\left(R_{p}, a\right)$, the fraction of planets with radius $R_{p}$ that are dense enough to be rocky, where $a$ denotes all the model parameters. Neglecting selection effects, the likelihood of finding the observed sample of planet M-R measurements given a model for the underlying population, is simply the probability that either both the planet is rocky (based on its M-R measurement) and that a planet of that size is rocky based on the population model or that both the planet is non-rocky (based on its M-R measurement) and a planet of that size would be non-rocky based on the population model.

$$
p(\text { data } \mid a)=\prod_{\text {planets }}\left\{p_{\text {rocky }} f_{\text {rocky }}+\left(1-p_{\text {rocky }}\right)\left(1-f_{\text {rocky }}\right)\right\}
$$

We then apply Bayes theorem, $p(a \mid$ data $) \propto p$ (data $\mid a) p(a)$, to calculated the posterior probability of the model parameters $p(a \mid$ data $)$ from the likelihood and the priors on the model parameters $p(a)$.

For illustration, we consider a 1-parameter step-function model for $f_{\text {rocky }}\left(R_{p}, a\right)$ where all planets larger than a threshold radius $R_{\text {thresh }}$ are non-rocky, while all planets smaller than $R_{\text {thresh }}$ are dense enough to be comprised of iron and silicates alone. Evaluating the posterior probability distribution of $R_{\text {thresh }}$ conditioned on the planet mass-radius data in Figure 1, we find a median value $R_{\text {thresh }}=1.73_{-0.05}^{+0.16} R_{\oplus}$, and a $95 \%$ confidence upper bound of $R_{\mathrm{thresh}}=1.83_{-0.04}^{+0.30} R_{\oplus}$. The $1 \sigma$ uncertainties quoted on the percentiles of $R_{\text {thresh }}$ were evaluated from 1000 bootstrapping samples. For the pure silicate composition adopted as the low-density extreme for rocky planets, the median and $95 \%$ upper bound on $R_{\text {thresh }}$ correspond to threshold masses of $5.8 M_{\oplus}$ and $7.2 M_{\oplus}$, respectively.

We have also investigated a 2-parameter linear model for $f_{\text {rocky }}\left(R_{p}, a\right)$, where all 
planets smaller than $R_{\text {min,non-rocky }}$ are dense enough to be rocky, all planets larger than $R_{\text {max } \text {,rocky }}$ are non-rocky, and where $f_{\text {rocky }}$ decreases linearly for $R_{\text {min,non-rocky }} \leqslant$ $R_{p} \leqslant R_{\text {max } \text {,rocky }}$. This linear transition model reduces to the step-function model when $R_{\text {min,non-rocky }}$ and $R_{\text {max,rocky }}$ are equal, but admits the possibility of a range of radii over which rocky and non-rocky planets coexist. The joint posterior probability density of $R_{\text {min,non-rocky }}$ and $R_{\text {max,rocky }}$ conditioned on the observed planets, sets a $2 \sigma$ upper limit on the maximum radii of rocky planets of $R_{\max \text {,rocky }} \leqslant 3.12 R_{\oplus}$, and a $2 \sigma$ upper limit on the planet radius at which no more than $5 \%$ of planets are dense enough to be rocky of $1.88 R_{\oplus}$.

We find that the simpler 1-parameter step-function model for $f_{\text {rocky }}$ is preferred over the 2-parameter linear transition model from the Bayesian evidence. More planet massradius measurements with smaller uncertainties are needed to constrain structure of the rocky/non-rocky transition.

\section{Summary}

We have presented an updated planet mass-radius diagram (Figure 1). None of the planets known to date that are larger than $2 R_{\oplus}$ are dense enough to be rocky. The results of an extensive Kepler follow-up program including multiple Doppler measurements for 22 KOIs Marcy et al. (2013) will soon more than double the population of sub-Neptune sized planets that have mass constraints. This opens the possibility of applying a hierarchical Bayesian model to learn about the underlying population of planet compositions.

We outline a statistical approach to infer the fraction of planets that are dense enough to be rocky (as a function of planet radius). We apply this approach to the current sample of planets with constraints on both mass and radius, and infer that most planets larger than $1.9 R_{\oplus}$ are too low density to be comprised of Fe and silicates alone. The results of this demonstration should be interpreted with caution, however, because the current sample of planets with both mass and radius constraints is an inhomogeneous sample with complicated selection effects. We look forward to having a larger sample of sub-Neptune size planets with measured masses once the Marcy et al. (2013) results are released. The constraints derived on the radii above which most planets are too low density to be composed of iron and silicate alone, will provide a useful empirical constraint for planet formation theories. Furthermore, our results will allow us to use the the sub-sample of KOIs with RV follow-up to inform us about the masses and compositions of the rest of the sub-Neptune size KOIs for which there is no RV follow-up.

\section{Acknowledgements}

L.A.R. acknowledges NASA support through Hubble Fellowship grant \#HF-51313.01A. This research has made use of the Exoplanet Orbit Database and the Exoplanet Data Explorer at exoplanets.org.

\section{References}

Batalha, N. M., Rowe, J. F., Bryson, S. T., et al. 2013, ApJS, 204, 24

Borucki, W. J., Koch, D. G., Basri, G., et al. 2011, ApJ, 728, 117

Marcy, G. W., Isaacson, H., Rowe, J. F., et al. 2013, ApJ, in prep.

Rogers, L. A. \& Seager, S. 2010a, ApJ, 712, 974

-. 2010b, ApJ, 716, 1208

Seager, S., Kuchner, M., Hier-Majumder, C. A., \& Militzer, B. 2007, ApJ, 669, 1279

Wright, J. T., Fakhouri, O., Marcy, G. W., et al. 2011, PASP, 123, 412 


\section{Discussion}

REBEKAH DAWson: In his presentation, Johnathan Fortney mentioned Kepler-36, where the difference in mass accounted for which planet retains a gassy atmosphere. Is there a physical motivation for making the cut in radius, instead of mass?

LESLIE ROGERS: The main motivation for exploring the fraction of planets that are dense enough to be rocky as a function of radius instead of mass is due to the selection effects, which are expected to have a more pronounced effect on the distribution of measured planet radii at specified mass, than on the distribution of measured planet masses at specified radius. The Marcy et al. (2013) sample of planets were initially discovered from their transits in Kepler photometry; for a given planet mass, larger radius lower density planets would be preferentially detected and identified by Kepler as planet candidates. The Marcy et al. (2013) KOIs were then selected for radial velocity follow-up based on their radius, and observed in a mass-blind way. Thus at a given radius the Marcy et al. (2013) RV planet masses should sample the underlying mass distribution without strong biases. Another motivation for studying planet composition as function of radius, is that our results translate readily to mass predictions for the thousands of Kepler planet candidates without RV follow-up. It is true, though, that for comparing to planet formation and evolution models, planet mass would provide a more direct comparison.

SloANe WiKTOROWICZ: I have a question about the result that smaller planets tend to denser, because it looks like when you get below $1 R_{\oplus}$ the probability for rocky is approaching $50 \%$. Is that just a radial velocity upper limits?

LESLIE Rogers: Yes, those are cases where we just have a radial velocity upper limit on the planet mass. Our information about the masses and compositions for those small planets below $1 R_{\oplus}$ is dominated by our priors.

JONATHAN FORTNEY: In terms of $p_{\text {rocky }}$, how do you handle the boundary? For planets just smaller than a pure rock composition, one could imagine that a water component is possible with a high iron/rock ratio.

LESLIE Rogers: In this analysis, I've essentially been considering a question with a yes/no answer: is the planet dense enough to be rocky based on the pure silicate massradius relation, or not. I have repeated this analysis considering instead an Earth-like composition threshold ( $32 \% \mathrm{Fe}, 68 \%$ silicate by mass), and found that the result that most planets larger than $1.9 R_{\oplus}$ are not rocky still holds, but the mass that radius threshold corresponds to increases to $\sim 9 M_{\oplus}$. 